VoL. 67 (2003) [267-276]

\title{
THE WIELANDT SERIES OF METABELIAN GROUPS
}

\author{
C.J.T. WETHERELL
}

The Wielandt subgroup of a group is the intersection of the normalisers of its subnormal subgroups. It is non-trivial in any finite group and thus gives rise to a series whose length provides a measure of the complexity of the group's subnormal structure. In this paper results of Ormerod concerning the interplay between the Wielandt series and upper central series of metabelian $p$-groups, $p$ odd, are extended to the class of all odd order metabelian groups. These extensions are formulated in terms of a natural generalisation of the upper central series which arises from Casolo's strong Wielandt subgroup, the intersection of the centralisers of a group's nilpotent subnormal sections.

\section{INTRODUCTION}

The Wielandt subgroup of a group $G$, denoted $\omega(G)$, is the intersection of the normalisers of the subnormal subgroups of $G$, ie

$$
\omega(G)=\left\{g \in G \mid S^{g}=S \text { for all } S \bowtie G\right\}
$$

The Wielandt series of $G$ is then defined by

$$
\omega_{0}(G)=1 \text { and } \omega_{i+1}(G) / \omega_{i}(G)=\omega\left(G / \omega_{i}(G)\right) \text { for } i \geqslant 0 .
$$

The least $n$ for which $\omega_{n}(G)=G$, if it exists, is called the Wielandt length of $G$ and denoted $w l(G)$. For example a group has Wielandt length one if and only if it is a $T$ group, one in which every subnormal subgroup is normal, and in general the Wielandt subgroup of any group is a $T$-group.

In [13] Helmut Wielandt, for whom the subgroup and series are named, showed in particular that every finite group has non-trivial Wielandt subgroup, since it contains the socle. Therefore for such groups the concept of Wielandt length is well-defined. Since then there has been much interest in what influence Wielandt length has on other group invariants; for example, exact bounds on the nilpotent length [3] and derived length [2] of finite soluble groups are known.

Received 5th September, 2002

I am deeply grateful for the guidance of my Ph.D. supervisor, John Cossey. This research was supported by an Australian Postgraduate Award.

Copyright Clearance Centre, Inc. Serial-fee code: 0004-9727/03 \$A2.00+0.00. 
The way in which Wielandt length bounds the nilpotency class $c(G)$ of a nilpotent group $G$ is less clear, although significant progress in this direction has been made by Ormerod in the articles $[6,7,8,9,10]$. In particular for odd primes $p$ the class of metabelian $p$-groups is well understood.

Theorem 1.1. (Ormerod [8].) Let $p$ be an odd prime and $P$ a finite metabelian p-group. Then $\omega_{n}(P) \leqslant \zeta_{n+1}(P)$ for all $n \geqslant 0$, and in particular $c(P) \leqslant w l(P)+1$.

If $w l(P)=1$ then $P$ is Dedekind and $c(P)=1$, but for larger Wielandt length Ormerod has shown in [6] that there exists a metacyclic $p$-group which attains the bound in Theorem 1.1 .

In this paper we shall extend Theorem 1.1 to the class of all metabelian groups of odd order. To account for non-nilpotent groups we require a generalisation of the upper central series due to Casolo [4].

The strong Wielandt subgroup of a group $G$, denoted $\bar{\omega}(G)$, is the intersection of the centralisers of the nilpotent subnormal sections of $G$, ie

$$
\bar{\omega}(G)=\left\{g \in G \mid[S, g] \leqslant S^{\mathfrak{N}} \text { for all } S \triangleleft \triangleleft G\right\}
$$

(Here $S^{\mathfrak{N}}$ is the nilpotent residual of $S$, the unique smallest normal subgroup $N$ of $S$ such that $S / N$ is nilpotent.) The strong Wielandt series, $\bar{\omega}_{i}(G)$, and strong Wielandt length, $\overline{w l}(G)$, are then defined in an analogous way to the Wielandt series and length.

Casolo established in [4] that the strong Wielandt subgroup of any finite group is non-trivial, since it too contains the socle, and so for such a group the concept of strong Wielandt length is well-defined. Notice that in a nilpotent group the strong Wielandt subgroup is equal to the centre, and therefore the strong Wielandt series and strong Wielandt length coincide with the upper central series and nilpotency class respectively.

We are able to extend Theorem 1.1 as follows.

THEOREM 2.4. Let $G$ be a metabelian group of odd order. Then for all $n \geqslant 0$ the section $\omega_{n}(G) / \bar{\omega}_{n}(G)$ is central and consequently $\omega_{n}(G) \leqslant \bar{\omega}_{n+1}(G)$. In particular $\overline{w l}(G) \leqslant w l(G)+1$ and this bound is best possible.

Whether this result extends further to the class of all finite metabelian groups depends, of course, on whether Theorem 1.1 applies to metabelian 2-groups, a problem which appears to be extremely difficult. Some partial results for even order metabelian groups, based on Schenkman's embedding of the Kern [11], will be discussed in Section 3.

A key result in this paper, which enables us to reduce the proof of Theorem 2.4 to the nilpotent case, is the fact that the strong Wielandt series of a subdirect product of metabelian groups can be easily determined from that of its subdirect factors; in fact we need only assume the factors have $p$-length 1 for all primes $p$. To make the precise statement of this result we employ the following notation. 
Notation. For an abstract subgroup function $\alpha$ and group $G$ with normal subgroup $N$, we denote by $\alpha(G \div N)$ the normal subgroup $A$ of $G$ such that $A / N=\alpha(G / N)$. For example $\omega\left(G \div \omega_{i}(G)\right)=\omega_{i+1}(G)$.

THEOREM 2.2. Let $G$ be a finite group with $p$-length 1 for all primes $p$ and normal subgroups $N_{1}$ and $N_{2}$. Then for any $n \geqslant 0$

$$
\bar{\omega}_{n}\left(G \div N_{1}\right) \cap \bar{\omega}_{n}\left(G \div N_{2}\right)=\bar{\omega}_{n}\left(G \div\left(N_{1} \cap N_{2}\right)\right) .
$$

In Section 4 we construct an example which shows that the $p$-length 1 hypothesis cannot be dropped.

\section{PROOFS}

In [2] Bryce and Cossey introduced a local version of the Wielandt subgroup, and in [4] Casolo mimicked this construction for the strong Wielandt subgroup. We begin with these important definitions. Recall that, for a set of primes $\pi$, a group $G$ is said to be $\pi$-perfect if it has no non-trivial $\pi$-quotients, ie $\mathrm{O}^{\pi}(G)=G$.

Let $G$ be a group and $p$ a prime. Then the $p$-Wielandt subgroup $\omega^{p}(G)$ of $G$ is the intersection of the normalisers of the $p^{\prime}$-perfect subnormal subgroups of $G$, and the $p$ strong Wielandt subgroup $\bar{\omega}^{p}(G)$ of $G$ is the intersection of the centralisers of the nilpotent quotients of $p^{\prime}$-perfect subnormal subgroups of $G$. That is

$$
\begin{aligned}
\omega^{p}(G) & =\left\{g \in G \mid S^{g}=S \text { for all } p^{\prime} \text {-perfect } S \triangleleft \triangleleft G\right\} \\
\text { and } \quad \bar{\omega}^{p}(G) & =\left\{g \in G \mid[S, g] \leqslant S^{\mathfrak{N}} \text { for all } p^{\prime} \text {-perfect } S \triangleleft \triangleleft G\right\}
\end{aligned}
$$

Our first lemma collects together some important properties of the Wielandt and strong Wielandt subgroups and their local counterparts. Most of these results appear in [2] or [4].

Lemma 2.1. Let $G$ be a finite group, $p$ a prime and $\sigma$ the set of prime divisors of $|G|$. Then

(a) $\langle\zeta(G)$, Soc $G\rangle \leqslant \bar{\omega}(G) \leqslant \omega(G)$, and in particular $w l(G) \leqslant \overline{w l}(G)$;

(b) $\omega(G)=\bigcap_{q \in \sigma} \omega^{q}(G)$ and $\bar{\omega}(G)=\bigcap_{q \in \sigma} \bar{\omega}^{q}(G)$;

(c) $\mathrm{O}_{p^{\prime}}(G) \leqslant \bar{\omega}^{p}(G) \leqslant \omega^{p}(G)$;

(d) $\omega^{p}(G)=\omega\left(G \div \mathrm{O}_{p^{\prime}}(G)\right)$ and $\bar{\omega}^{p}(G)=\bar{\omega}\left(G \div \mathrm{O}_{p^{\prime}}(G)\right)$;

(e) if $K$ is subnormal in $G$ with $p^{\prime}$-index then $\omega^{p}(G) \cap K=\omega^{p}(K)$ and $\bar{\omega}^{p}(G)$ $\mathrm{n} K=\bar{\omega}^{p}(K)$.

(f) if $H$ is a normal Hall subgroup of $G$ then $\omega_{n}(G) \cap H=\omega_{n}(H)$ and $\bar{\omega}_{n}(G)$ $\cap H=\bar{\omega}_{n}(H)$ for all $n \geqslant 0$.

Suppose further that $G$ is soluble, then 
(g) $\bar{\omega}(G) \leqslant \zeta($ Fit $G)$ and in particular $\bar{\omega}(G)$ is Abelian;

(h) if $\mathrm{O}_{p^{\prime}}(G)=1$ and $\omega(G)$ is non-nilpotent then Fit $G \leqslant \omega(G)$;

(i) $[\omega(G), G] \leqslant$ Fit $\omega(G)$.

Proof: (a)-(e) See [2, 3.7,3.2 and 3.8] and [4, Proposition 1, Lemmas 2 and 3].

(f) The case $n=1$ was first proved for the Wielandt series in [1]. A shorter proof of this case, which adapts more readily to the strong Wielandt series, is included here.

Let $\pi$ be the set of prime divisors of $|H|$. If $p \in \pi$ then $H$ has $p^{\prime}$-index in $G$ and so $\omega^{p}(G) \cap H=\omega^{p}(H)$ by (d). On the other hand if $q \notin \pi$ then $H \leqslant \mathrm{O}_{q^{\prime}}(G) \leqslant \omega^{q}(G)$ by (c). Applying (b) gives

$$
\omega(G) \cap H=\bigcap_{q \notin \pi}\left(\omega^{q}(G) \cap H\right) \cap \bigcap_{p \in \pi}\left(\omega^{p}(G) \cap H\right)=H \cap \bigcap_{p \in \pi} \omega^{p}(H)=\omega(H)
$$

and the case $n=1$ is established.

Now let $n>1$ and assume the result holds for $n-1$. Set $W=\omega_{n-1}(G)$. By the case $n=1$ and the modular law we have

$$
\left(\omega_{n}(G) \cap H\right) W / W=\omega(G / W) \cap H W / W=\omega(H W / W),
$$

since $H W / W$ is the unique Hall $\pi$-subgroup of $G / W$. Via the natural isomorphism $H W / W \rightarrow H /(H \cap W)$ we see that

$$
\left(\omega_{n}(G) \cap H\right) /(H \cap W)=\omega(H /(H \cap W))
$$

and the result follows at once since $H \cap W=\omega_{n-1}(H)$ by our inductive assumption. The proof for the strong Wielandt series is identical.

(g) Set $F=$ Fit $G$, then $[\bar{\omega}(G), F] \leqslant F^{\mathfrak{N}}=1$ and $\bar{\omega}(G) \leqslant \mathrm{C}_{G}(F)=\zeta(F)$.

(h), (i) See [2, 3.4 and 3.10].

The important result concerning subdirect products of metabelian groups is proved next.

Theorem 2.2. Let $G$ be a finite group with p-length 1 for all primes $p$ and normal subgroups $N_{1}$ and $N_{2}$. Then for any $n \geqslant 0$

$$
\bar{\omega}_{n}\left(G \div N_{1}\right) \cap \bar{\omega}_{n}\left(G \div N_{2}\right)=\bar{\omega}_{n}\left(G \div\left(N_{1} \cap N_{2}\right)\right) .
$$

Proof: By induction it suffices to establish that the case $n=1$ holds. Suppose otherwise and choose $G, N_{1}, N_{2}$ and $H \triangleleft G$ all of minimal order subject to

$$
H \leqslant \bar{\omega}\left(G \div N_{1}\right) \cap \bar{\omega}\left(G \div N_{2}\right) \text { but } H \nless \bar{\omega}\left(G \div\left(N_{1} \cap N_{2}\right)\right) \text {. }
$$

Clearly $N_{1} \cap N_{2}=1$ by the minimality of $|G|$. Since each $\bar{\omega}\left(G / N_{i}\right)$ is Abelian it follows, from the minimality of $|H|$, that $H$ is an Abelian $p$-group for some prime $p$. For every 
$q \neq p$ we have $H \leqslant \mathrm{O}_{q^{\prime}}(G) \leqslant \bar{\omega}^{q}(G)$ and so $H \nless \bar{\omega}^{p}(G)$ by Lemma $2.1(\mathrm{~b})$. Thus there exists a $p^{\prime}$-perfect subnormal subgroup $S$ such that $[S, H] \nless S^{\mathfrak{N}}$. For any $T \triangleleft \triangleleft H S$ and each $i=1,2$ we have

$$
[H, T] \leqslant T^{\mathfrak{n}} N_{i} \cap H S=T^{\mathfrak{N}}\left(N_{i} \cap H S\right)
$$

and therefore $H \leqslant \bar{\omega}\left(H S \div\left(N_{i} \cap H S\right)\right)$. If $G \neq H S$ then minimality of $|G|$ gives $H \leqslant \bar{\omega}(H S)$ and $[S, H] \leqslant S^{\mathfrak{N}}$, thus $G=H S$. In particular $G$ is $p^{\prime}$-perfect and therefore $p$-nilpotent, having $p$-length 1 by hypothesis.

Set $K=\mathrm{O}_{p^{\prime}}(G)$. For every $T \triangleleft \triangleleft G$ and each $i$ we have

$$
[T, H] \leqslant T^{\mathfrak{N}} N_{i} \cap H K=T^{\mathfrak{N}}\left(N_{i} \cap H K\right),
$$

since $T^{\mathfrak{N}} \leqslant G^{\mathfrak{N}}=K$, and therefore $H \leqslant \bar{\omega}\left(G \div\left(N_{i} \cap H K\right)\right)$. The minimality of $\left|N_{i}\right|$ shows that we must have $N_{i} \leqslant H K$. In particular $N_{i} K=\mathrm{O}_{p}\left(N_{i}\right) \times K$ and so $N_{1} K \cap N_{2} K=K$. Clearly $H K / K \leqslant \bar{\omega}\left(G / K \div N_{i} K / K\right)$ for each $i$, thus if $K \neq 1$ minimality of $|G|$ and Lemma 2.1(d) give the contradiction

$$
H K / K \leqslant \bar{\omega}(G / K)=\bar{\omega}^{p}(G) / K .
$$

Therefore $K=1$ and in fact $G$ is a $p$-group. Now

$$
[H, G] \leqslant G^{\mathfrak{M}} N_{1} \cap G^{\mathfrak{N}} N_{2}=N_{1} \cap N_{2}=1
$$

and we conclude that $H \leqslant \zeta(G)=\bar{\omega}(G)$, a final contradiction. This establishes that $\bigcap_{i} \bar{\omega}\left(G \div N_{i}\right) \leqslant \bar{\omega}\left(G \div \bigcap_{i} N_{i}\right)$, and the reverse inclusion follows easily from the definitions.

An easy corollary is that groups in this class with bounded strong Wielandt length constitute a formation.

COROLLARY 2.3. For any integer $n$, the class of finite groups with p-length 1 for all primes $p$ and strong Wielandt length at most $n$ is a formation.

This further justifies regarding the strong Wielandt series as a natural generalisation of the upper central series, since the formation property is true of nilpotent groups with bounded nilpotency class. In fact for a group $G$ with $p$-length 1 for all $p$ there is also a natural generalisation of the lower central series: let $\bar{\omega}_{0}^{*}(G)=G$ and for $i \geqslant 0$ define

$$
\bar{\omega}_{i+1}^{*}(G)=\bigcap\left\{N \triangleleft G \mid \bar{\omega}_{i}^{*}(G) \leqslant \bar{\omega}(G \div N)\right\} .
$$

Then $\bar{\omega}_{i}^{*}(G) \leqslant G_{i}$ for any normal series

$$
G=G_{0} \geqslant G_{1} \geqslant \cdots \geqslant G_{k}=1,
$$

with each $G_{i} / G_{i+1} \leqslant \bar{\omega}\left(G / G_{i+1}\right)$, and moreover $\left\{\bar{\omega}_{i}^{*}(G)\right\}$ is itself such a series. In particular this lower series has shortest possible length, equal to the strong Wielandt 
length of $G$, and it coincides with the lower central series when $G$ is nilpotent. (In fact the above construction works equally well for many other well-known abstract subgroup functions and classes of groups. See Chapter 4 of [12] for an introductory investigation into the general question of when a canonical lower series exists.)

We are now in a position to prove the main result. The reader is referred to [5, Section $A, 12]$ for standard results on coprime actions.

THEOREM 2.4. Let $G$ be a metabelian group of odd order. Then for all $n \geqslant 0$ the section $\omega_{n}(G) / \bar{\omega}_{n}(G)$ is central and consequently $\omega_{n}(G) \leqslant \bar{\omega}_{n+1}(G)$. In particular $\overline{w l}(G) \leqslant w l(G)+1$ and this bound is best possible.

Proof: Suppose the first claim is false and that $G$ and $n>0$ are minimal such that $\left[\omega_{n}(G), G\right] \nless \bar{\omega}_{n}(G)$. If $G$ has distinct minimal normal subgroups $M_{1}$ and $M_{2}$ then by minimality of $|G|$ and Theorem 2.2 we have

$$
\left[\omega_{n}(G), G\right] \leqslant \bigcap_{i}\left[\omega_{n}\left(G \div M_{i}\right), G\right] \leqslant \bigcap_{i} \bar{\omega}_{n}\left(G \div M_{i}\right)=\bar{\omega}_{n}(G),
$$

a contradiction. Thus $G$ has unique minimal normal subgroup $M$, say, and if $p$ is the prime divisor of $|M|$ it follows that $\mathrm{O}_{p^{\prime}}(G)=1$. In particular $G$ has a unique Sylow p-subgroup $P=$ Fit $G$, and $G / P$ is Abelian.

Set $R=G^{\mathfrak{N}}, W=\omega(G)$ and $V=\bar{\omega}(G)$, noting that $R \neq 1$ by Theorem 1.1 and $V=\zeta(P)$ by parts (f) and (g) of Lemma 2.1. If $P$ is Abelian then

$$
\left[\omega_{n}(G), G\right] \leqslant G^{\prime} \leqslant P=V \leqslant \bar{\omega}_{n}(G),
$$

thus we may assume $P$ is non-abelian and therefore not contained in the $T$-group $W$. By Lemma 2.1(h) $W$ must be nilpotent and in fact $W=\omega(P)$ by Lemma 2.1(f). We show next that $W \cap R=V$.

Since $R$ is Abelian it is complemented in $G$, by [5, IV,5.18], thus we can find a complement $H$, say, to $R$ in $P$. Now $W=\omega(P)$ normalises $H$ and it follows that

$$
[W \cap R, P]=[W \cap R, R H] \leqslant R^{\prime}[W \cap R, H] \leqslant R \cap H=1,
$$

that is $W \cap R \leqslant \zeta(P)=V$.

Let $Q$ be a Hall $p^{\prime}$-subgroup of $G$. Then $G / R=P / R \times Q R / R$ and it follows that $R=(Q R)^{\mathfrak{N}}$ and $R=[R, Q R]=[R, Q]$. Now $R$, being non-trivial, contains $M$ and therefore $M=[M, Q]$. In particular $M$ is non-central and we must have $\zeta(G)=1$. Notice next that $G / \mathrm{C}_{G}(V)$ is a $p^{\prime}$-group and so $V$ has the decomposition

$$
V=\mathrm{C}_{V}(G) \times[V, G] .
$$

One of these factors must be trivial, since $G$ has a unique minimal normal subgroup, yet if $V=\mathrm{C}_{V}(G)$ then $V \leqslant \zeta(G)=1$. Therefore $V=[V, G]$ and

$$
V=[V, G]=[V, G, \ldots, G] \leqslant[G, G, \ldots, G]=G^{\mathfrak{N}}=R,
$$


for sufficiently large weight. This establishes the equality $W \cap R=V$.

Since $Q$ is an Abelian $p^{\prime}$-group of operators for $P$ we have

$$
[G, Q]=[P Q, Q]=[P, Q]=[P, Q, \ldots, Q] \leqslant R
$$

Moreover

$$
\omega_{n}(G)=\left(\omega_{n}(G) \cap P\right)\left(\omega_{n}(G) \cap Q\right) \leqslant \omega_{n}(P) Q,
$$

via Lemma 2.1(f), and it follows that

$$
\left[\omega_{n}(G), G\right] \leqslant\left[\omega_{n}(P) Q, P Q\right] \leqslant\left[\omega_{n}(P), P\right][G, Q] \leqslant\left[\omega_{n}(P), P\right] R .
$$

Appealing to Theorem 1.1 and the fact that $\zeta(P)=V \leqslant R$ we have

$$
\left[\omega_{n}(G), G\right] \leqslant\left[\omega_{n}(P), P\right] R \leqslant \zeta_{n}(P) R \leqslant \zeta_{n-1}(P \div R) \leqslant \bar{\omega}_{n-1}(G \div R) .
$$

On the other hand minimality of $n$ shows that

$$
\left[\omega_{n}(G), G\right]=\left[\omega_{n-1}(G \div W), G\right] \leqslant \bar{\omega}_{n-1}(G \div W),
$$

and by Theorem 2.2 we have

$$
\left[\omega_{n}(G), G\right] \leqslant \bar{\omega}_{n-1}(G \div(W \cap R))=\bar{\omega}_{n-1}(G \div V)=\bar{\omega}_{n}(G),
$$

a final contradiction.

It follows that

$$
\omega_{n}(G) \leqslant \zeta\left(G \div \bar{\omega}_{n}(G)\right) \leqslant \bar{\omega}\left(G \div \bar{\omega}_{n}(G)\right)=\bar{\omega}_{n+1}(G)
$$

and, when $n=w l(G)$, that $\overline{w l}(G) \leqslant w l(G)+1$. Any non-abelian soluble $T$-group establishes that this bound is best possible for $w l(G)=1$, and for $w l(G) \geqslant 2$ we appeal to Ormerod's work [6] on the Wielandt series of metacyclic $p$-groups.

In [10] Ormerod considered metabelian $p$-groups in which the Wielandt subgroup is either as small or as large as possible, and what restrictions these conditions place on higher terms of the Wielandt series. One of the main results is

Theorem 2.5. (Ormerod [10].) Let $p$ be an odd prime and $P$ a finite metabelian p-group. If $\omega(G)=\zeta_{2}(G)$ then $\omega_{n}(G)=\zeta_{n+1}(G)$ for all $n \geqslant 1$, and if $\omega_{2}(G)=\zeta_{2}(G)$ then $\omega_{n}(G)=\zeta_{n}(G)$ for all $n \geqslant 2$.

As an immediate corollary to Theorem 2.4 we see that the first statement of Theorem 2.5 generalises to all odd order metabelian groups.

CoROLlary 2.6. Let $G$ be a metabelian group of odd order. If $\omega(G)=\bar{\omega}_{2}(G)$ then $\omega_{n}(G)=\bar{\omega}_{n+1}(G)$ for all $n \geqslant 1$ and in particular $G / \bar{\omega}(G)$ is nilpotent. 
We have been unable to decide whether the second statement of Theorem 2.5 also generalises. We might attempt to modify the proof of Theorem 2.4 but two problems arise: it is not immediately apparent that a minimal counter-example will have unique minimal normal subgroup since the condition $\omega_{2}(G)=\bar{\omega}_{2}(G)$ is not preserved by quotients; $G / \omega(G)$ has Wielandt and strong Wielandt subgroups which coincide, yet Ormerod has shown in Section 3 of [10] that in general this places very little restriction on higher terms of the two series, thus the inductive argument on the quotients $G / \omega(G)$ and $G / G^{\mathfrak{N}}$ is not so fruitful in this setting.

\section{MEtabelian gROUPS WITH EVEN ORDER}

By the nature of its proof we can see that the conclusion of Theorem 2.4 applies to all finite metabelian groups if and only if it applies to finite metabelian 2-groups. (There is only one place in the proof, other than appealing to Theorem 1.1, where we have assumed the prime $p$ is odd: that $P$ is not a $T$-group if it is non-abelian and therefore, via Lemma $2.1(\mathrm{~h})$, that $W$ must be nilpotent. The same argument does not work when $p=2$ since $P$ might be Hamiltonian. However $W$ is already nilpotent in this case since it is 2-nilpotent, being a $T$-group and hence supersoluble, and $\mathrm{O}_{2^{\prime}}(G)=1$.)

While metabelian 2-groups are far from understood in this context, we do know that the Wielandt subgroup of any nilpotent group is contained in the second centre: Schenkman proved in [11] that the Kern of a group, the intersection of normalisers of all subgroups, is contained in the second centre, but of course in a nilpotent group the Kern and Wielandt subgroup coincide since all subgroups are subnormal. Thus we can easily adapt the proof of Theorem 2.4 to find partial results for metabelian groups with even order; indeed the proof relies only on the fact that $G^{\mathfrak{N}}$ is Abelian, since we do not need to assume the Sylow subgroups are metabelian to apply Schenkman's result.

Theorem 3.1. Let $G$ be a finite Abelian-by-nilpotent group. Then $\omega(G) / \bar{\omega}(G)$ is central and in particular $\omega(G) \leqslant \bar{\omega}_{2}(G)$.

Finally we note that Schenkman's result for nilpotent groups can also be extended to the wider class of groups with $p$-length 1 for all $p$, although for such a group $G$ we may lose the centrality of the section $\omega(G) / \bar{\omega}(G)$.

TheOREM 3.2. Let $G$ be a finite group with $p$-length 1 for all primes $p$. Then $\omega(G) \leqslant \bar{\omega}_{2}(G)$.

Proof: Let $G$ be a minimal counter-example, then it has a unique minimal normal subgroup so that $\mathrm{O}_{p^{\prime}}(G)=1$ for some prime $p$ and $P=$ Fit $G$ is the unique Sylow $p-$ subgroup of $G$. Applying parts (f), (h) and (i) of Lemma 2.1 shows that $\omega(G)$ must be nilpotent, since $\omega(G) P / P$ is central and consequently $P \neq \bar{\omega}(G)=\zeta(P)$. Therefore $\omega(G)=\omega(P) \leqslant \zeta_{2}(P) \leqslant \bar{\omega}_{2}(G)$, a contradiction. 


\section{EXAmples}

We begin with an example which establishes that Theorem 3.1 is best possible in the sense that, even for a nilpotent-by-abelian group, the Wielandt subgroup need not be central modulo the strong Wielandt subgroup. Let $L=S L_{2}(3)$, the special linear group of degree 2 over $G F(3)$ with generators $i=\left(\begin{array}{ll}0 & 1 \\ 2 & 0\end{array}\right)$ and $j=\left(\begin{array}{ll}2 & 1 \\ 1 & 1\end{array}\right)$ of order 4 and $a=\left(\begin{array}{ll}1 & 1 \\ 0 & 1\end{array}\right)$ of order 3 . Then $L$ has normal Sylow 2-subgroup $P=\langle i, j\rangle$ isomorphic with the quaternions of order 8 . Moreover $\mathrm{O}_{2^{\prime}}(L)=1$ and, since $\omega(L)$ must therefore be nilpotent, $\omega(L)=\omega(P)=P$ and $\bar{\omega}(S)=\zeta(P)=\left\langle i^{2}\right\rangle$ by Lemma 2.1(f). However

$$
[\omega(L), L] \ni[i, a]=i j \notin \bar{\omega}(L) .
$$

Next we show that the $p$-length 1 condition is necessary for the crucial intersection property of Theorem 2.2. Let $Q=\langle x, y\rangle$ be quaternion of order 8 and define an action of $Q$ on $L$ by

$$
i^{x}=j, j^{x}=i, a^{x}=a^{2},[L, y]=1 .
$$

Form the semi-direct product $G=L \rtimes Q$, noting that $G$ has 2-length equal to 2. Since $i^{2}=j^{2}$ and $x^{2}=y^{2}$ are both central in $G$ the subgroups $N_{1}=\left\langle y^{2}\right\rangle$ and $N_{2}=\left\langle i^{2} y^{2}\right\rangle$ are normal in $G$. Clearly $N_{1} \cap N_{2}=1$.

Notice next that $[G, L]=L$ since $a=\{a, x]$ and $L=\left\langle a, L^{\prime}\right\rangle$. Since $G / L$ is nilpotent we have $L=G^{\mathfrak{N}}$. If $M_{1}=\langle x, L\rangle$ and $M_{2}=\langle x y, L\rangle$ then for the same reasons $L=M_{1}^{\mathfrak{N}}$ $=M_{2}^{\mathfrak{N}}$. All other subnormal subgroups of $G$ are contained in $\langle y, L\rangle$ and are therefore centralised by $y$. On the other hand $[G, y]=\left\langle y^{2}\right\rangle$ and

$$
L \not \nexists y^{2} \in L N_{1}=L N_{2} .
$$

It follows therefore that $y \in \bar{\omega}\left(G \div N_{1}\right) \cap \bar{\omega}\left(G \div N_{2}\right)$ but $y \notin \bar{\omega}(G)$.

Note however that the group $G$ is not the subdirect product of two groups with strictly smaller strong Wielandt length (this is because its strong Wielandt length and derived length coincide). Indeed we have been unable to construct an example of such a group, thus the formation property of Corollary 2.3 might hold in larger classes of groups than just those with $p$-length 1 for all primes $p$.

\section{REFERENCES}

[1] A. Ali, 'On the Wielandt length of a finite supersoluble group', Proc. Roy. Soc. Edinburgh Sect. A 130 (2000), 1217-1226.

[2] R.A. Bryce and J. Cossey, 'The Wielandt subgroup of a finite soluble group', J. London Math. Soc. (2) 40 (1989), 244-256.

[3] A.R. Camina, 'The Wielandt length of finite groups', J. Algebra 15 (1970), 142-148. 
[4] C. Casolo, 'Wielandt series and defects of subnormal subgroups in finite soluble groups', Rend. Sem. Mat. Univ. Padova 87 (1992), 93-104.

[5] K. Doerk and T.O. Hawkes, Finite soluble groups (Walter de Gruyter, Berlin, 1992).

[6] E.A. Ormerod, 'The Wielandt subgroup of metacyclic p-groups', Bull. Austral. Math. Soc. 42 (1990), 499-510.

[7] E.A. Ormerod, 'Groups of Wielandt length two', Math. Proc. Cambridge Philos. Soc. 110 (1991), 229-244.

[8] E.A. Ormerod, 'On the Wielandt length of metabelian p-groups', Arch. Math. (Basel) 57 (1991), 212-215.

[9] E.A. Ormerod, 'Some p-groups of Wielandt length three', Bull. Austral. Math. Soc. 58 (1998), 121-136.

[10] E.A. Ormerod, 'A note on the Wielandt subgroup of a metabelian p-group', Comm. Algebra 27 (1999), 621-627.

[11] E. Schenkman, 'On the norm of a group', Ilinois J. Math. 4 (1960), 150-152.

[12] C.J.T. Wetherell, Subnormal structure of finite soluble groups, (Ph.D. thesis) (Australian National University, ACT, Australia, 2001). http://thesis.anu.edu.au.

[13] H. Wielandt, 'Über den Normalisator der subnormalen Untergruppen', Math. Z. 69 (1958), 463-465.

Mathematical Sciences Institute

ANU

Canberra, ACT 0200

Australia 